\title{
Anti-Hepatitis E Antibody in Hemodialysis Patients in Isfahan, Iran: Prevalence and Risk Factors
}

\author{
Seyed Moayed Alavian, ${ }^{1}$ Behrooz Ataei, ${ }^{2,}$ Ali Ebrahimi, ${ }^{3}$ Omid Pirhaji, ${ }^{3}$ Roya Azad, ${ }^{3}$ Behrooz \\ Olya, ${ }^{3}$ and Amir Mohammad Ataei ${ }^{3}$ \\ ${ }^{1}$ Baqiyatallah Research Center for Gastroenterology and Liver Diseases, Baqiyatallah University of Medical Sciences, Tehran, IR Iran \\ ${ }^{2}$ Infectious Diseases and Tropical Medicine Research Center, Isfahan University of Medical Sciences, Isfahan, IR Iran \\ ${ }^{3}$ Medical Students' Research Center, Isfahan University of Medical Sciences, Isfahan, IR Iran \\ ${ }^{*}$ Corresponding Author: Behrooz Ataei, Infectious Diseases and Tropical Medicine Research Center, Isfahan University of Medical Sciences, Isfahan, IR Iran. Tel: +98-3113377172, \\ E-mail: Ataei@med.mui.ac.ir
}

Received: September 14, 2014; Revised: February 11, 2015; Accepted: March 4, 2015

\begin{abstract}
Background: Many studies have been performed regarding the prevalence of hepatitis E in the general population, but there is controversial evidence for an increased risk of the infection in patients on maintenance hemodialysis (HD).

Objectives: The primary end point of the present study was to determine if the prevalence of anti-hepatitis E virus IgG (anti-HEV IgG) is higher in patients on maintenance HD than in the normal population in Isfahan.

Patients and Methods: In a case-control study performed in Isfahan in June 2012, we compared the seroprevalence of HEV among 274 patients on maintenance HD and 275 otherwise healthy individuals. The patients were recruited from $3 \mathrm{HD}$ centers in Isfahan. Anti-HEV IgG was detected using a Dia Pro Diagnostic HEV enzyme immunoassay(ELISA) kit. Demographic and clinical data (sex, age, blood transfusion history, HD duration, age at HD initiation, and evidence of hepatitis B and hepatitis C infections) were obtained from the medical records of the HD patients.

Results: Anti-HEV IgG was detected in 27 (9.9\%) controls and 78 (28.3\%) patients, with the difference being statistically significant $(\mathrm{P}<0.05)$. Furthermore, there was a significant association between positive anti-HEV antibody, HD duration, and blood transfusion history in the HD patients.

Conclusions: Considering the results, it seems necessary to conduct prospective studies in order to identify factors responsible for the high seroprevalence of HEV in Isfahan HD units.
\end{abstract}

Keywords: Hemodialysis; Hepatitis E; Prevalence; Serology

\section{Background}

Hepatitis E virus (HEV) is a single-strand, non-enveloped RNA virus. It is the only member of the genus Hepevirus in the Hepeviridae family. HEV is responsible for acute hepatitis epidemics in developing countries with suboptimal sanitation and sporadic hepatitis in developed countries (1-3). The major route of HEV transmission is fecal-oral through contaminated water (4). However, some studies have suggested parenteral transmission as an important route of the disease transmission, especially in hemodialysis (HD) patients and patients with a history of blood transfusion $(1,5)$. HEV has worldwide distribution, but tropical climates, inadequate sanitation, and poor personal hygiene are factors that can cause higher prevalence in some regions (6). The prevalence of seropositive patients ranges from $15 \%$ to $60 \%$ in endemic regions (2). Asia, Africa, Middle East, and Central America have the highest incidence of HEV infection. Iran is also among the countries having experienced periods of HEV outbreaks in the past (1). Different seroprevalence of HEV has been reported in different cities of Iran, ranging from $3.8 \%$ in Isfahan to $11.5 \%$ in Khuzestan $(7,8)$.

Chronic HD patients are known to be at high risk for infection due to their immunocompromised condition. Also, frequent hospital contacts expose these patients to different infectious agents (9). Although HEV usually causes a self-limited acute infection with a generally low mortality rate, acute severe liver disease and fulminant hepatitis can occur (10).

\section{Objectives}

To date, different studies have reported controversial results about the seroprevalence of HEV in HD patients (11). Herein, we report our experience vis-a-vis anti-HEV IgG seroprevalence in a selected population of HD patients in Isfahan.

\section{Patients and Methods}

This case-control study, performed in June 2012, recruited 274 patients on maintenance HD from 3 HD centers

Copyright (C) 2015, Kowsar Corp. This is an open-access article distributed under the terms of the Creative Commons Attribution-NonCommercial 4.0 International License (http://creativecommons.org/licenses/by-nc/4.0/) which permits copy and redistribute the material just in noncommercial usages, provided the original work is properly cited. 
Alavian SM et al.

in Isfahan Province. Additionally, 275 otherwise healthy individuals who were receiving no medication were recruited from the same geographic location. The study was conducted with the approval of the scientific and ethics committee of Isfahan University of Medical Sciences. All the participants signed a written informed consent form. Tests for the HEV serum marker (anti-HEV IgG) were carried out using a commercially available enzyme-linked immunosorbent assay kit (Dia Pro Diagnostic, Italy). The patients were split into 2 groups according to their anti-HEV status (i.e. HEV positive or negative). The tests were conducted in accordance with the manufacturer's instruction. Medical records were reviewed, and information on age, sex, HD duration, age at HD initiation, history of blood transfusion, and evidence of hepatitis $\mathrm{B}$ or hepatitis $\mathrm{C}$ infection was collected.

Statistical analysis was performed using Statistical Package for the Social Sciences (SPSS) for Windows (version 16.0, 2007, SPSS Inc. Chicago, IL, USA). The Student t-test, analysis of variance, $\chi^{2}$ test, and Fisher exact test were used to compare the variables. Furthermore, to avoid possible bias, we employed multivariate logistic regression analysis. A P value $<0.05$ was considered statistically significant.

\section{Results}

The study population comprised 274 patients on maintenance HD (145 males and 129 females) and 275 healthy controls (140 males and 135 females). Gender was significantly different between the 2 groups $(\mathrm{P}=0.03)$. The mean age of the HD and non-HD subjects was $59.9 \pm 16.4$ and $46.6 \pm 18.2$ years, respectively. (Age range was 21 - 80 years in the patients and 24 - 71 in the healthy individuals). The mean duration ( \pm Standard Deviation [SD]) of HD treatment was 34.60 \pm 13.70 months, ranging from 8 to 86 months. The mean $( \pm$ SD) age at HD initiation was $49 \pm 13.6$ years. Diabetes mellitus was the underlying disease in 85 patients, followed by hypertension (72), glomerulonephritis (39), polycystic kidney disease (17), nephrolithiasis (15), urological disease (10), and other causes (5); the cause could not be ascertained in the remaining cases. Eighty-four patients had a history of blood transfusion. No one was infected with HBV or hepatitis $\mathrm{C}$ virus (HCV). Among the studied individuals, the ELISA results demonstrated the existence of anti-HEV IgG antibody in $78(28.3 \%)$ patients and 27 (9.9\%) healthy controls; the difference was statistically significant $(P<0.001)$. The frequency distribution of HEV in the HD and non-HD subjects is depicted in Table 1 . The results showed a significant association between a positive anti-HEV antibody result and blood transfusion history, duration of dialysis, age at HD initiation, and sex among the HD patients. There were no statistically significant associations between the other risk factors (i.e. HBV and HCV infection, age, and underlying cause of renal failure) and HEV infection. A comparison of the patient characteristics between the anti-HEV IgG seropositive and seronegative groups is presented in Table 2.

Multivariate logistic regression analysis was applied to calculate the adjusted odds ratio for the potential risk factors for HEV infection among the HD patients; it demonstrated that blood transfusion history and duration of dialysis were significantly associated with HEV infection. The results are depicted in Table 3.

\begin{tabular}{|c|c|c|c|c|c|c|}
\hline \multirow{3}{*}{$\begin{array}{l}\text { HEV } \\
\text { Positive }\end{array}$} & \multicolumn{4}{|c|}{ Groups ${ }^{c}$} & \multirow{2}{*}{\multicolumn{2}{|c|}{ Total }} \\
\hline & \multicolumn{2}{|c|}{ HD Patients } & \multicolumn{2}{|c|}{ Non-HD Patients } & & \\
\hline & 78 & $(28.3)$ & 27 & (9.9) & 105 & $(19.1)$ \\
\hline Negative & 196 & (71.7) & 247 & (90.1) & 445 & (80.9) \\
\hline Total & 274 & (100) & 274 & (100) & 550 & (100) \\
\hline \multicolumn{7}{|c|}{$\begin{array}{l}\text { a Values are presented as No. (\%). } \\
\text { b Abbreviations: HEV, hepatitis E virus; HD, hemodialysis. } \\
\text { c } \mathrm{P}<0.001 .\end{array}$} \\
\hline \multirow{2}{*}{\multicolumn{7}{|c|}{$\begin{array}{l}\text { Variable } \\
\text { Gender }\end{array}$}} \\
\hline & & & & & & \\
\hline \multicolumn{2}{|l|}{ Male } & $33(22.8)$ & & $112(77.2)$ & \multicolumn{2}{|c|}{0.03} \\
\hline \multicolumn{2}{|l|}{ Female } & $45(34.6)$ & & $85(65.4)$ & & \\
\hline \multicolumn{7}{|c|}{ Blood Transfusion History } \\
\hline \multicolumn{2}{|l|}{ Yes } & $35(41.7)$ & & $49(58.3)$ & \multicolumn{2}{|c|}{0.001} \\
\hline \multicolumn{2}{|l|}{ No } & $42(22.7)$ & & $143(77.3)$ & & \\
\hline \multicolumn{2}{|l|}{ Age, $y$} & $58.16 \pm 2.09$ & & $61.32 \pm 1.14$ & \multicolumn{2}{|c|}{0.46} \\
\hline \multicolumn{2}{|c|}{ Age at dialysis initiation, $y$} & $52.75 \pm 2.19$ & & $59.36 \pm 1.17$ & \multicolumn{2}{|c|}{0.02} \\
\hline Duration c & on & $62.92 \pm 4.69$ & & $21.18 \pm 1.63$ & \multicolumn{2}{|c|}{$<0.001$} \\
\hline
\end{tabular}

a Values are presented as mean \pm SD or No. (\%).

b Abbreviations: HEV, hepatitis E virus; HD, hemodialysis. 
Alavian SM et al.

\begin{tabular}{|c|c|c|}
\hline Variable & OR & 95\% CI \\
\hline \multicolumn{3}{|l|}{ Gender } \\
\hline Female & 1.88 & $0.81-4.35$ \\
\hline Male & 1 & - \\
\hline \multicolumn{3}{|l|}{ Underlying Cause of Renal Failure } \\
\hline Diabetes & 8.64 & $0.87-86.28$ \\
\hline Hypertension & 3.48 & $0.34-35.78$ \\
\hline Glomerulonephritis & 6.41 & $0.13-318.10$ \\
\hline Polycystic kidney disease & 8.10 & $0.49-133.80$ \\
\hline Urological disease & 11.09 & $0.68-179.65$ \\
\hline Other causes & 29.52 & $0.34-257.5$ \\
\hline Idiopathic & 5.20 & $0.30-89.53$ \\
\hline \multicolumn{3}{|l|}{ Blood Transfusion } \\
\hline Yes & 4.02 & $1.15-14.01^{b}$ \\
\hline No & 1 & - \\
\hline Age & 0.88 & $0.58-1.35$ \\
\hline Age at dialysis initiation & 1.12 & $0.73-1.70$ \\
\hline Duration of dialysis & 1.06 & $1.02-1.11^{\mathrm{C}}$ \\
\hline Blood transfusion $\times$ Duration of dialysis $d$ & 0.98 & $0.95-1.01$ \\
\hline
\end{tabular}

\section{Discussion}

The present study was conducted to determine the prevalence of HEV infection among patients on chronic HD and to evaluate whether chronic HD is associated with higher risk of the infection. The study population was comprised of 274 HD patients and 275 non-HD controls. The HEV antibody was detected in $78 / 275$ (28.3\%) of the HD patients and in $27 / 274$ (9.9\%) of the control group.

HEV is a causative agent of non-A, non-B hepatitis. HEV is mainly transmitted through the fecal-oral route, but parenteral and vertical routes have also been described. Therefore, there may be an increased risk of HEV infection in HD patients with an amplified risk of exposure to blood-transmitted agents insofar as a few studies have reported outbreaks of HEV infection in HD patients $(12,13)$.

The diagnosis of HEV infection is usually made using serological markers in HD patients. However, the infection can also be detected by finding the genome in serum or stool via polymerase chain reaction. The IgM antibody is detected in the initial phase of the infection right before the peak of alanine aminotransferase (ALT) activity and gradually decreases within weeks and months after ALT normalization. For anti-HEV IgG, different studies have reported a persistence range of 6 months to 14 years. Since the response of the IgG antibody to HEV weakens after the acute phase of infection, it seems that this an- tibody is mostly useful to detect acute infection. Both anti-HEV IgM and anti-HEV IgG antibodies are associated with false-positive and false-negative results. Moreover, a significant level of the IgG antibody is cleared during the process of hemodialysis. Therefore, using antibody markers to detect HEV infection is accompanied by some shortcomings, especially in HD patients (14).

Data concerning the seroprevalence of HEV infection among HD patients have presented conflicting results. The HEV seropositivity rates reported in HD patients for different countries range as low as $0.9 \%$ in France and $2.6 \%$ in Italy and as high as $7.3 \%$ or more among the adult population in Spain or in Brazil (14). A case-control study using serology assays conducted in Saudi Arabia reported a prevalence of $4.8 \%$ in 83 chronic HD patients vs. $0.3 \%$ in healthy controls. In that study, the HD patients had a significantly higher prevalence of HEV infection than did the control group (15). Psichogiou et al. detected anti-HEV IgG in $6.4 \%$ of the HD patients and $2.2 \%$ of the reference group in their study (16). To the best of our knowledge, our study represents the highest seroprevalence of HEV among HD patients to date. Nonetheless, Kikuchi et al. reported a high seroprevalence of HEV (i.e. 19\%) among their HD patients, which is approximately concordant with our results (17). 
Although the prevalence of HEV infection was far more common in our study, the infection was more prevalent in the HD patients, which chimes in with the results of the aforementioned studies. In contrast to these results, Taremi et al. reported a lower prevalence of infection in HD patients vs. the general population (7.4\% vs. 9.6\%) (11).

Hosseini-Moghaddam et al. hypothesized that the seroprevalence of HEV in patient groups, such as HD patients, is dependent on the prevalence of HEV infection in the general population (1). Meanwhile, our other study does not support this hypothesis even when considering a previous general population-based study performed in Isfahan (7).

The higher prevalence of anti-HEV IgG antibody in our study could be due to lower public health, poor health strategies in HD units, or past local HEV infection in Isfahan. Moreover, genetic variations of HEV in different geographic locations and false-positive results caused by other infections may constitute other possible explanations for such differences in the results.

The association between anti-HEV positivity and potential risk factors such as sex, age, blood transfusion history, hepatitis B or hepatitis C serology, and HD duration has been evaluated in some studies. Psichogiou et al. reported no significant association between anti-HEV and underlying renal disease; anti-HCV, anti-hepatitis B core antibody; blood transfusions; and history of elevated transaminases, clinical hepatitis, and renal transplantation. Nevertheless, the authors found a marginal association with HD duration in their univariate analysis, which was not confirmed in their multivariate analysis (16). In a study performed in Greece, no association was found between anti-HEV positivity and age or sex, duration of $\mathrm{HD}$, hepatitis B or C virus infection serology, previously elevated aminotransferase levels, and transfusion history (12). In another study performed in Turkey, no significant association was determined between anti-HEV IgG positivity and HD duration, blood transfusion history, and anti-HCV and HBsAg positivity (18).

The association between HEV seropositivity and HD duration, demonstrated in the present study, is in accordance with some previous studies (19). Anti-HEV antibody seropositivity increases significantly with age, from less than 10\% among individuals between 6 and 19 years of age to more than $40 \%$ among those aged more than 60 years (20). A study performed in Iran reported a seroprevalence of $1.2 \%$ in children under 10 years old and $7.3 \%$ in subjects aged between 20 and 25 years (21). However, the results of our study did not reveal a significant difference in age between the anti-HEV-positive and anti-HEV-negative subjects. This could be due to the narrow range of the study patients' age.

Although HEV has traditionally been believed to be a selflimited acute infection that infrequently becomes chronic, more recent articles have reported chronic hepatitis, and even cirrhosis, caused due to HEV in organ transplantation recipients $(22,23)$. Furthermore, HEV-related chronic hepatitis has been reported in HIV patients and among individuals on rituximab treatment for non-Hodgkin lymphoma (24-26). On the other hand, HEV usually causes a self-limited acute infection with a generally low mortality rate, but there is a likelihood of the occurrence of acute severe liver disease and fulminant hepatitis (10).

In light of the aforementioned evidence, immunosuppression could be the cause of HEV chronicity. Also, there is risk of fulminant hepatitis in HEV infection. Therefore, the high prevalence of HEV in the immune compromised HD patients in the current study may be a serious warning.

Our study has some limitations that warrant due attention. Firstly, the control group was not matched for age and sex with the cases. Secondly, the presumptive diagnosis of HEV infection was based on the antibody assay, which is not gold standard especially in HD patients. Thirdly, the study subjects were not tested for anti-HEV IgM. Accordingly, we cannot arrive at a conclusion concerning exposure time in the seropositive subjects.

In sum, our study revealed a high prevalence of HEV infection among HD patients, which was significantly more prevalent than that in the general population. Moreover, the study showed that blood transfusion history and HD duration could be potential risk factors for HEV infection. With respect to the high prevalence of infection in Isfahan HD units, it seems necessary to adopt appropriate strategies to find intra-unit factors causing the high prevalence of HEV serology markers. Furthermore, it seems essential that further studies be performed to clarify risk factors associated with HEV infection in HD patients.

\section{Authors' Contributions}

All the authors were involved in study design, data collection, data analysis, and manuscript preparation.

\section{Funding/Support}

The study was financially supported by The National Foundation of Elites.

\section{References}

1. Hosseini-Moghaddam SM, Zarei A, Alavian SM, Mansouri M Hepatitis E virus infection: a general review with a focus on hemodialysis and kidney transplant patients. Am J Nephrol. 2010;31(5):398-407.

2. Dong C, Dai X, Liang J, Dong M, Meng J. Seroprevalence of hepatitis e virus varies considerably among chinese provinces. Hepat Mon. 2012;12(6):386-90.

3. Mushahwar IK. Hepatitis E virus: molecular virology, clinical features, diagnosis, transmission, epidemiology, and prevention. $J$ Med Virol. 2008;80(4):646-58.

4. Kheradpezhouh M, Taremi M, Gachkar L, Aghabozorgi S, Khoshbaten $M$. Presence and significance of transfusion-transmitted virus infection in Iranian patients on maintenance hemodialysis. J Microbiol Immunol Infect. 2007;40(2):106-11.

5. Rodríguez-Frias F, Jardi R, Buti M, Hepatitis E. Molecular virology, epidemiology and pathogenesis. Medicina Clínica. 2012;138(2, 4):69-72.

6. Zhuang H, Cao XY, Liu CB, Wang GM. Epidemiology of hepatitis E in China. Gastroenterol Jpn. 1991;26 Suppl 3:135-8.

7. Ataei B, Nokhodian Z, Javadi AA, Kassaian N, Shoaei P, Farajzadegan Z, et al. Hepatitis E virus in Isfahan Province: a populationbased study. Int J Infect Dis. 2009;13(1):67-71. 
8. Assarehzadegan MA, Shakerinejad G, Amini A, Rezaee SA. Seroprevalence of hepatitis E virus in blood donors in Khuzestan Province, southwest Iran. Int J Infect Dis. 2008;12(4):387-90.

9. Horl WH. Neutrophil function and infections in uremia. Am J Kidney Dis. 1999;33(2):xlv-xlviii.

10. Beladi Mousavi SS, Motemednia F, Beladi Mousavi M. Epidemiology of hepatitis e virus infection in patients on chronic hemodialysis. Jundishapur J Microbiol. 2014;7(5):e6993.

11. Taremi M, Khoshbaten M, Gachkar L, EhsaniArdakani M, Zali M. Hepatitis E virus infection in hemodialysis patients: a seroepidemiological survey in Iran. BMC Infect Dis. 2005;5:36.

12. Stefanidis I, Zervou EK, Rizos C, Syrganis C, Patsidis E, Kyriakopoulos $\mathrm{G}$, et al. Hepatitis E virus antibodies in hemodialysis patients: an epidemiological survey in central Greece. Int J Artif Organs. 2004;27(10):842-7.

13. Pisanti FA, Coppola A, Galli C. Association between hepatitis C and hepatitis E viruses in southern Italy. Lancet. 1994;344(8924):746-7.

14. Zekavat OR, Makarem A, Karami MY, Amanat A, Mohandes M, Habibagahi M. Serological investigation for hepatitis E virus infection in the patients with chronic maintenance hemodialysis from southwest of Iran. Asian J Transfus Sci. 2013;7(1):21-5.

15. Ayoola EA, Want MA, Gadour MO, Al-Hazmi MH, Hamza MK. Hepatitis $\mathrm{E}$ virus infection in haemodialysis patients: a case-control study in Saudi Arabia. JMed Virol. 2002;66(3):329-34.

16. Psichogiou M, Vaindirli E, Tzala E, Voudiclari S, Boletis J, Vosnidis G, et al. Hepatitis E virus (HEV) infection in haemodialysis patients. The Multicentre Haemodialysis Cohort Study on Viral Hepatitis. Nephrol Dial Transplant. 1996;11(6):1093-5.

17. Kikuchi K, Yoshida T, Kimata N, Sato C, Akiba T. Prevalence of hepatitis E virus infection in regular hemodialysis patients. Ther
Apher Dial. 2006;10(2):193-7.

18. Ucar E, Cetin M, Kuvandik C, Helvaci MR, Gullu M, Huzmeli C. [Hepatitis E virus seropositivity in hemodialysis patients in Hatay province, Turkey]. Mikrobiyol Bul. 2009;43(2):299-302.

19. Pourahmad M, Sotoodeh AR, Nasiri N. Hepatitis E virus infection in hemodialysis patients: a seroepidemiological survey in Jahrom, Southern Iran. Hepat Mon. 2009;9(3):232-5.

20. Thomas DL, Yarbough PO, Vlahov D, Tsarev SA, Nelson KE, Saah AJ, et al. Seroreactivity to hepatitis E virus in areas where the disease is not endemic. J Clin Microbiol. 1997;35(5):1244-7.

21. Saffar MJ, Farhadi R, Ajami A, Khalilian AR, Babamahmodi F, Saffar H. Seroepidemiology of hepatitis E virus infection in 2-25-year-olds in Sari district, Islamic Republic of Iran. East Mediterr Health J. 2009;15(1):136-42.

22. Rostamzadeh Khameneh Z, Sepehrvand N, Masudi S. Seroprevalence of hepatitis E among Iranian renal transplant recipients. Hepat Mon. 2011;11(8):646-51.

23. Haagsma EB, van den Berg AP, Porte RJ, Benne CA, Vennema $\mathrm{H}$, Reimerink JH, et al. Chronic hepatitis E virus infection in liver transplant recipients. Liver Transpl. 2008;14(4):547-53.

24. Puoti M, Moioli MC, Travi G, Rossotti R. The burden of liver disease in human immunodeficiency virus-infected patients. Semin Liver Dis. 2012;32(2):103-13.

25. Dalton HR, Bendall RP, Keane FE, Tedder RS, Ijaz S. Persistent carriage of hepatitis $\mathrm{E}$ virus in patients with HIV infection. $N$ Engl J Med. 2009;361(10):1025-7.

26. Ollier L, Tieulie N, Sanderson F, Heudier P, Giordanengo V, Fuzibet JG, et al. Chronic hepatitis after hepatitis E virus infection in a patient with non-Hodgkin lymphoma taking rituximab. Ann Intern Med. 2009;150(6):430-1. 